\title{
COMMITTEE II: Guidelines for Cytologic Sampling Techniques of Lung and Mediastinal Lymph Nodes.
}

\author{
Michael $\mathbf{C W}^{1}$, Faquin $\mathbf{W}^{2}$, Jing $\mathbf{X}^{3}$, Kaszuba $\mathrm{F}^{4,}$ Kazakov $\mathbf{J}^{5}$, Moon \\ $E^{6}$, Toloza $\mathrm{E}^{7}, \mathrm{Wu} \mathrm{RI}^{8}$, and Moreira AL.
}

1. Department of Pathology and Laboratory Medicine, University Hospitals Cleveland Medical Center/Case Western Reserve University, Cleveland, OH

2. Department of pathology, Massachusetts General Hospital and Harvard Medical School, Boston, MA

3. Department of Pathology, University of Michigan, Ann Arbor, MI

4. Division of Pulmonary, Critical Care \& Sleep Medicine, H. Lee Moffitt Cancer Center/University of South Florida, Tampa, FL

5. Department of Internal Medicine, University Hospitals Cleveland Medical Center/Case Western Reserve University, Cleveland, $\mathrm{OH}$

6. Department of Internal Medicine, University of Pennsylvania Health System and Perelman School of Medicine, Philadelphia, PA

7. Department of Thoracic Oncology, H. Lee Moffitt Cancer Center/ University of south Florida, Tampa, FL

8. Department of Pathology and Laboratory Medicine, University of Pennsylvania Health system and Perelman School of Medicine, Philadelphia, PA.

9. Department of Pathology, New York University Langone Health, New York. N.Y

This is the author manuscript accepted for publication and has undergone full peer review but has not been through the copyediting, typesetting, pagination and proofreading process, which may lead to differences between this version and the Version of Record. Please cite this article as doi: $10.1002 / \mathrm{dc} .23975$

This article is protected by copyright. All rights reserved. 


\section{Corresponding Author:}

Claire W. Michael, M.D.

Department of Pathology

University Hospitals Cleveland Medical Center/Case Western Reserve University

11100 Euclid Ave Rm 212B

Cleveland, OH 44106

Phone: 216-844-0137

Fax: 216-844-1810

Email: claire.michael@uhhospitals.org

This article is protected by copyright. All rights reserved. 


\begin{abstract}
The Papanicolaou Society of Cytopathology has developed a set of guidelines for pulmonary cytology including indications for bronchial brushings, washings and endobronchial ultrasound guided transbronchial fine needle aspiration (EBUS-TBNA), technical recommendations for cytological sampling, recommended terminology and classification schemes, recommendations for ancillary testing and recommendations for post-cytological management and follow-up. All recommendations are based on the expertise of the authors, an extensive literature review and feedback from presentations at national and international conferences. This document selectively presents the results of these discussions.

The present document summarizes recommendations regarding techniques used to obtain cytological and small histologic specimens from the lung and mediastinal lymph nodes including rapid on site evaluation (ROSE), and the triage of specimens for immunocytochemical and molecular studies.
\end{abstract}

\title{
Key Words
}

Clinical Evaluation, Imaging Studies, Techniques for Cytological Study Including Bronchial Washings, bronchial Brushings, and Endobronchial Ultrasound Guided Transbronchial Fine Needle Aspiration, Rapid On Site Evaluation, Specimen Triage, Papanicolaou Society of Cytopathology Recommendations for Pulmonary Cytology 


\section{Introduction}

In an effort to standardize respiratory cytology practice, the Papanicolaou Society of Cytopathology (PSC) developed a set of guidelines. The standardized terminology and nomenclature for respiratory cytology have recently been published, ${ }^{1}$ along with the consensus recommendations for the utilization of ancillary studies for diagnosis. ${ }^{2}$ The following is Committee II report on guidelines for techniques utilized for respiratory sampling that complements the previously published Committee I report on the guidelines for indications of such sampling techniques. ${ }^{3}$

\section{Exfoliative Cytology Sampling Techniques}

\section{A. Sputum}

\section{i. Spontaneous Sputum}

Spontaneous sputum is the simplest method of collecting a respiratory specimen for cytological examination. Its reported sensitivity in the literature ranges from $42 \%$ to $97 \%$ and specificity ranges from $68 \%$ to $100 \%$, primarily as a result of lack of standardized programs for its collection and processing among laboratories, with a pooled sensitivity of $66 \%$ and specificity of $99 \%$. Spontaneous sputum samples have a sensitivity of $71 \%$ for central tumors versus $49 \%$ for peripheral tumors. ${ }^{4}$ Although it has a lower sensitivity relative to other methods, it still has a role in the diagnosis of patients with suspected lung masses and is recommended as the initial test in patients who present with central lesions with or without metastasis and those who are at high risk for semi-invasive procedures. ${ }^{4}$ Despite its lower sensitivity, sputum is a noninvasive, simple and cost effective method that is consequently used particularly in countries with limited resources.

The patient is instructed to cough deeply and to expel the contents into a sterile container. A morning pooled sample is ideal when looking for cancer, ${ }^{5}$ and the diagnostic rate is proportional to the number of samples examined. The optimal number is three samples for the diagnosis of cancer and five samples to exclude cancer. ${ }^{6}$ While diagnostic accuracy approaches $95 \%$ when five sequential samples are submitted, ${ }^{7}$ additional samples are not contributory. ${ }^{8}$ In cases of tuberculosis, examination of three early morning sputum samples on consecutive days approaches the sensitivity of a single sputum specimen submitted for culture. ${ }^{9}$ The specimen should be stored at $4^{\circ} \mathrm{C}$ and transferred to the lab immediately for processing. However, in cases

of bacterial culture, results were unaffected by storage at room temperature for up to 6 hours, and could be stored up to 48 hours at $4{ }^{\circ} \mathrm{C} .{ }^{10}$ Prolonged exposure to ambient temperature does not adversely affect the yield of samples for acid-fast bacilli. Unfortunately, a review of studies assessing macroscopic and/or microscopic sputum quality and the impact of pre-sputum collection interventions did not result in definitive conclusions due to the paucity and heterogeneity of available data. ${ }^{11}$

Several studies have shown that collecting sputum in liquid based cytology (LBC) collecting media such as CytoLyte ${ }^{\circledR}$ or CytoRich red ${ }^{\circledR}$ and subjecting the sputum to homogenization during the liquid based preparation resulted in more cellular samples, better quality and higher 
sensitivity with less unsatisfactory and atypical diagnosis when compared to the traditional pick and smear method of preparation. This processing techniques is particularly useful in evaluating sputums collected from patients with suspected lung cancer. A complementary cell block improved the sensitivity and allowed the performance of immunohistochemistry and acceptable DNA extraction for molecular testing. ${ }^{12-14}$

\section{ii. Induced Sputum Collection}

Induced sputum provides better quality specimens and better diagnostic yield in small lesions than spontaneous sputum. ${ }^{15}$ In the setting of possible infection, attempting induction early, before starting antibiotics, may increase diagnostic yield. ${ }^{16}$ While some authors have recommended mouth rinsing, spitting out saliva, using nose clips, and/or brushing teeth prior to sputum collection, the value of these procedures is unclear and may add unnecessary complexity to the protocol. ${ }^{17}$ The technical aspects are as follows: bronchodilator $(200 \mu \mathrm{g}$ inhaled salbutamol) is administered to prevent bronchospasm; patient breathes saline (0.9 to 6\%) through nebulizer for 15-30 minutes, patient is asked to cough or spit every five minutes or whenever they have the urge to do so, and FEV1 should be measured at the end of each induction interval. $^{17}$

In cases submitted to microbiology, no statistically significant differences were found between the yield of induced and spontaneous sputum samples. ${ }^{18}$ Although sputum induction may provide an adequate sample more frequently than spontaneous sputum, it is more costly and may not result in more patients starting treatment for mycobacterial infection. ${ }^{19}$ Yield for acidfast bacilli smears and culture increases with repeated sputum induction. ${ }^{20}$ However, a mix of unsupervised, supervised, and induced sputum may be more practical than three induced sputum specimens. $^{21}$ In a subset of patients, chest physiotherapy for bronchial clearance may be helpful as an alternative method for obtaining sputum. ${ }^{22}$

While induced sputum should be processed within two hours, examination of cell counts can be delayed for up to nine hours with refrigeration at $4{ }^{\circ} \mathrm{C} .{ }^{23}$ Induced sputum samples collected from patients with suspected cancer can be processed by liquid based preparations as described above.

\section{iii. Post-Bronchoscope Sputum (PBS)}

A post-bronchoscopy sputum smear may be helpful in combination with bronchoalveolar lavage particularly for the detection of pulmonary tuberculosis (TB) in patients with suspected pulmonary TB who had either three negative sputum smears or could not expectorate. ${ }^{24}$ Postbronchoscopy sputum analysis can increase diagnostic yield in patients who are smear negative or non-productive of sputum. ${ }^{25,26}$

Once transbronchial exfoliative samples are retrieved, the patient is placed in the supine position while the selector catheter is in position and $10 \mathrm{~mL}$ of sterile saline are rapidly injected. The catheter is then withdrawn and the patient turned on his side with the affected lung being uppermost. The patient is then instructed to cough into a container. ${ }^{27}$ alternatively patients could be asked to provide a sputum sample if they become productive in the hours following the bronchoscopy procedure. ${ }^{25}$ If the specimen is collected for microbiological testing, the specimen 
needs to be fresh for special stains and cultures. Sputum specimens sent to the cytology laboratory should be handled similarly to the other sputum samples as described above.

\section{B. Specimens Collected During Bronchoscopy}

\section{i. Bronchial Brush}

If a brush is indicated, a Teflon tube is inserted with a nylon brush mounted within the end of the tube. The brush is then advanced beyond the tube over the suspected area and moved back and forth vigorously. The goal is to brush the lesion directly and entrap bits of the lesion on the bristles. ${ }^{28}$ The brush is then partially pulled back into the Teflon tube and both are withdrawn. The sample is consequently submitted according to the laboratory policies, which may include immediate smear preparation and alcohol fixation and/or rinsing in saline or other preservatives. This technique demonstrates better yield when a central visible lesion is targeted, as opposed to submucosal or peripheral lesions. If multiple areas are to be sampled and a tumor is recognized, it should be brushed last to prevent cross-contamination by cancer cells. ${ }^{29}$

\section{ii. Bronchial Wash and Bronchoalveolar Lavage (BAL)}

Once the tracheobronchial tree is inspected, the bronchoscope is wedged into the selected bronchus. Normal saline at room temperature with total volume of $100-300 \mathrm{~mL}$ is instilled through the bronchoscope, divided into three to five aliquots. Suction pressure less than $100 \mathrm{~mm}$ $\mathrm{Hg}$ or low enough to prevent visible airway collapse is applied. Optimal sampling should retrieve $\geq 30 \%$ of the instilled volume. The procedure should be stopped if less than $5 \%$ of the instilled aliquot is recovered. ${ }^{30,31}$ Alternatively, a bronchial wash may be used to sample the airway surface if a lesion is identified. Smaller volumes of room temperature normal saline are instilled $(5-50 \mathrm{~mL})$. The smaller volumes may be beneficial when performing this diagnostic procedure on a marginally stable patient.

For both bronchial wash and bronchoalveolar lavage, $5-15 \mathrm{~mL}$ of fluid are required for basic microbiologic analysis and 10-20 $\mathrm{ml}$ are needed for cellular analysis such as cell counts and/or biomarkers for interstitial lung disease. The collected specimens should be kept at room temperature if immediate transportation and processing are expected. If a delay of 30-60 min is anticipated, the specimens should be transported on ice $\left(4^{\circ} \mathrm{C}\right)$. If a delay more than $60 \mathrm{~min}$ is expected, the specimen should be centrifuged and resuspended in nutrient-supplemented medium (MEM or RPMI). The resuspended specimen can be stored at $\left(4^{\circ} \mathrm{C}\right)$ for 24 hours. If centrifugation is not available, MEM or RPMI could be added to the pooled lavage and the specimen can be stored at $\left(4^{\circ} \mathrm{C}\right)$ for up to 12 hours. ${ }^{30}$ Again, for cytological evaluation, the sample can be collected directly into one of the LBC collecting media.

Warming the $0.9 \%$ sterile saline to $37^{\circ} \mathrm{C}$ may reduce cough and bronchospasm, but most use saline at room temperature. ${ }^{32}$ Suctioning under low pressure $(25-100 \mathrm{mmHg})$ avoids collapse of distal airways and trauma to the mucosal surface. ${ }^{32}$ There is variation in the volume of fluid used for bronchoalveolar lavage, ranging from 100-300 mL in each lung segment or subsegment sampled; smaller volumes are more likely dominated by a "bronchial" washing component. ${ }^{32}$ There is also variation in the number of aliquots/doses over which the saline is infused. The 
bronchoscope should be maintained in the wedged position throughout the procedure to avoid contamination from the large airways. Dwell time should be minimized.

The right middle lobe (RML) or lingula are standard sites for BAL sampling, unless there are localized findings on imaging. ${ }^{32}$ The RML is associated with highest volume of return for nontargeted BAL. ${ }^{33}$ Yield is generally better for central rather than peripheral lesions. Additional material can be captured from a guide sheath flush, which may have a higher diagnostic yield than bronchoalveolar lavage. ${ }^{34}$

There is no data to support sending the first aliquot for microbiology and the subsequent aliquots for cellular analysis versus pooling all the aliquots. ${ }^{35}$ The volume of fluid recovered as well as the percent of fluid returned should be reported for the pooled specimen to ensure adequate recovery. ${ }^{36}$ Lavage fluid should be stored in silicone-coated or similar containers to prevent macrophage adhesion. ${ }^{36}$

\section{Transbronchial Sampling Techniques}

\section{A. Endobronchial Ultrasound Guided Sampling}

\section{i. Background}

Endobronchial ultrasound (EBUS) is a bronchoscopic technique, first described in 2006, that uses ultrasound technology to visualize structures within the airway wall, lung, mediastinum, and hila. ${ }^{37-39}$ Two types of EBUS exist: radial probe EBUS (RP-EBUS / equipped with a 20-30MHz probe) and convex probe EBUS (CP-EBUS / equipped with a 7.5MHz probe). For the purposes of this guideline, "EBUS" refers to the CP-EBUS type which is the one relevant for mediastinal/hilar nodal biopsy. There are multiple published reviews incorporating a comprehensive discussion of indications, contraindications, and potential complications of EBUS bronchoscopy. This section will focus for the approach of EBUS sampling of hilar and mediastinal lymph nodes for staging of lung cancer.

EBUS has an established role in the diagnosis and staging of patients with lung cancer and is able to access more nodal stations than the traditional approach of cervical mediastinoscopy. ${ }^{40}$ Lymph node analysis is a critical piece of the tumor-node-metastasis (TNM) staging system that is standard for the work-up of patients with non-small cell lung cancer (NSCLC). EBUS should be the initial procedure for sampling mediastinal and hilar lymph nodes that are suspected to harbor loco-regional metastatic disease.

\section{ii. General Approach}

Patients who already have a diagnosis of lung cancer from tissue sampling of their primary lung lesion, but lack staging information, will often proceed to EBUS lymph node staging when multidisciplinary oncologic planning deems it appropriate. If there is a clinical suspicion of lung cancer, but no diagnostic tissue confirmation, attempts should be made to both diagnose and stage during the same bronchoscopic procedure. Multiple modalities, including transbronchial biopsies, brushings, and lavage, with advanced guidance strategies, like software navigation, ${ }^{41}$ 
electromagnetic navigation, ${ }^{42}$ and peripheral radial ultrasound, ${ }^{43}$ can be used to confirm the lung cancer diagnosis during the same EBUS staging procedure.

In general, the mediastinal and hilar lymph nodes accessible by EBUS include 2R, 2L, 3p, 4R, 4L, 7, 10R, 10L, 11R, and 11L (Fig. 1). EBUS cannot access paraesophageal lymph nodes, and while EBUS can access subaortic level 5-6, it tends to be less accurate and associated with more risks. ${ }^{44}$ To access those lymph nodes, EBUS can be done in conjunction with transesophageal endoscopy (EUS). ${ }^{45}$ Though there is data to support the use of ultrasound echotexture characteristics to differentiate between malignant and benign lymph nodes, ${ }^{46,47}$ lymph nodes that are $0.5 \mathrm{~cm}$ or greater in short-axis diameter on EBUS analysis should be considered for transbronchial needle aspiration (TBNA). Thorough evaluation of bilateral hila and mediastinum should be performed during the EBUS evaluation in light of the false-negative rate of nuclear and radiologic staging. ${ }^{48,49}$ EBUS-BNA of enlarged mediastinal/hilar lymph nodes should be performed in a systematic fashion, bearing in mind the laterality of the suspected primary lung lesion. For instance, if a patient presents with a spiculated right upper lobe pulmonary nodule and an enlarged subcarinal lymph node on CT evaluation, but does not yet have a confirmed diagnosis, the recommended approach is EBUS bronchoscopy with systematic scanning and TBNA of enlarged lymph nodes from the furthest contralateral lymph node space ("highest stage") to the closest ipsilateral lymph node space ("lowest stage"). This allows for use of a single EBUS needle for the whole procedure while minimizing the risk for contamination and false "up-staging" of the patient. Rapid on-site evaluation (ROSE) of aspirated material from the subcarinal lymph node (Fig. 2) should be performed as confirmation of diagnostic material which would obviate the need for biopsy attempts of the primary lesion, which carries a higher peri-procedural risk than EBUS-TBNA. If ROSE does not confirm diagnostic material, the bronchoscopist should consider transitioning to brushings, biopsies, and lavage of the lung nodule.

\section{iii. Technical Approach}

The bronchoscopist should remember the specifics of the airway view offered by the EBUS bronchoscope when intubating the patient and navigating the airways. The EBUS bronchoscope offers an $80^{\circ}$ visual field of view at a visual angle of $35^{\circ}$ forward oblique. The ultrasound view is parallel to the shaft of the bronchoscope, with an angle of $90^{\circ}$ (Fig. 3). The balloon attached to the probe can be gently inflated with saline to allow optimal apposition with the airway wall for best ultrasound viewing. Color flow and Doppler measurements can be performed to identify vascular and cystic structures. Caliper measurements can be performed on the ultrasound image to assess lymph node size.

In real-time with ultrasound viewing of an enlarged lymph node, a transbronchial needle ( 21 or $22 \mathrm{G}$ retractable sharp beveled needle with a stylet contained within a flexible catheter) can be inserted through the working channel of the bronchoscope. Once the catheter emerges from the bronchoscope, the needle is pushed through the airway wall and into the target lymph node under ultrasound visualization. Suction can be applied and the catheter is agitated 20-30 times per pass. Once sampling is complete, suction is released and the needle is pulled into the catheter and the whole system is removed from the bronchoscope. If the sample is being sent for cytological analysis without ROSE, the aspirated material should be expelled into a sterile sample cup containing a few milliliters of saline using a combination of saline/air flushes and stylet insertions through the needle or rinsed in LBC collecting media. If the sample is being 
evaluated by ROSE, the needle should be extended onto a slide and the stylet inserted gently through the needle until sufficient material has been expelled onto the slide. The remaining sample within the needle can be expelled into a sterile sample cup as above. An average of three passes per lymph node should be performed. ${ }^{50,51}$ There is data to support the utility of additional passes to obtain sufficient material for molecular analysis. ${ }^{52}$

Traditionally the $22 \mathrm{G}$ needle is chosen during EBUS bronchoscopy because the degree to which it stiffens the tip of the EBUS bronchoscope is less than that of the $21 \mathrm{G}$ needle. This is an important issue in lymph node stations where appropriate apposition may be suboptimal and the insertion of the larger $21 \mathrm{G}$ needle makes it even more difficult (e.g. the paratracheal spaces.) Some experts believe the $21 \mathrm{G}$ needle can produce "core-biopsy-like" material to work-up suspected lymphoma diagnoses or is able to acquire more tissue for molecular profiling of lung cancer cells. However, studies have produced conflicting results regarding specimen adequacy and diagnostic yield between the two needle sizes. ${ }^{53-55}$ There have been efforts to develop larger gauge needles and miniature forceps (to obtain transbronchial lymph node biopsies with preserved nodal architecture) appropriate for the EBUS bronchoscope, but further studies will need to be done to confirm their utility. A recent pilot study evaluating the Echo Tip ProCore needle ${ }^{\circledR}$ showed no additive value to specimen adequacy when compared with the conventional needle. ${ }^{56}$

Recently, new 19 G EBUS needle became available. Tyan et al. reported their experience with the new needle on 47 patients with hilar and mediastinal lymphadenopathy in three centers. The diagnostic yield for malignancy, sarcoidosis and reactive lymph node hyperplasia was 89\%, $93 \%$ and $83 \%$. All 13 patients diagnosed with adenocarcinoma by the $19 \mathrm{G}$ needle had sufficient tissue for genetic studies. Further studies and direct comparison with the $22 \mathrm{G}$ and $21 \mathrm{G}$ are needed to establish if the $19 \mathrm{G}$ needle is superior to the smaller gauge needles. ${ }^{57}$

\section{B. Endobronchial Sampling Techniques of Pulmonary Parenchymal Lesions}

Sampling techniques for pulmonary parenchymal lesions (PPLs) by endoscopic means may be performed via brushing, needle aspiration, forceps biopsies or any combination of the aforementioned techniques. Relatively newer techniques such as radial endobronchial ultrasound and navigational bronchoscopy or a combination of both procedures may add some diagnostic accuracy to the procedure. Overall diagnostic percentage yield percentage rates for routine bronchoscopic specimens range from the mid-teens to mid 80's depending on the lesion size $(<20 \mathrm{~mm}$ or $>20 \mathrm{~mm})$, location, the presence of an airway leading into the lesion and means of biopsy or combination of biopsy methods. The vast majority of the studies reviewed have focused on suspected lung cancer with the diagnostic yield in other malignancies or metastatic disease showing lower diagnostic yields. ${ }^{58}$

\section{i. Sampling of Parenchymal lesions by Endobronchial Brushings}

A variety of commercially available cytology brushes sharing similar characteristics of flexible bristles encased within a sterile single use sheath are available. Bristle diameters range from 1.2-3 mm with the length of the brush typically measuring $10 \mathrm{~mm}$. The tip is usually 
rounded and metallic for improved visualization while utilizing fluoroscopy. The procedure may be performed using moderate sedation or general anesthesia. After adequate sedation, the bronchoscope is advanced into the broncho-pulmonary segment of interest and then using either fluoroscopy or navigational guidance, the brush sheath is advanced to the leading edge of the lesion. The brush is then extruded from its sheath through the bronchoscope and advanced 1-3 $\mathrm{cm}$ into the area of interest followed by a back and forth motion for several seconds while visualized by fluoroscopy. The brush is then retracted into the sheath and removed from the bronchoscope, after which the tip can be immediately extruded and brushed onto a slide, which is then placed into fixative solution, or clipped into a sterile specimen cup with normal saline for processing at a later time. This process is then repeated between 2 and 6 times. Several techniques such as brush rotation and repeated brushing have been described, however, no specific technique has been shown to definitively increase yield or diagnostic accuracy. There is also no consensus on number of brushings that should be performed. One older study has shown that brushes with longer, wider bristles provided a greater yield in the number of cells per brush. ${ }^{59}$ It is generally accepted that performing brushings prior to biopsies is preferable due to decreased peripheral blood contamination.

\section{ii. Radial EBUS (R-EBUS) and Transbronchial Needle Aspiration (TBNA)}

Radial EBUS and navigational bronchoscopy are adjunctive technologies which provide the clinician additional directional and spatial information as to where to direct brushes and/or forceps to improve diagnostic accuracy. R-EBUS guidance is utilized to confirm accurate localization of a PPL prior to biopsying it. This is particularly useful when the PPL is in a peripheral location and requires navigation through multiple branching airway segments to reach it. When multiple branches need to be traversed, navigation bronchoscopy can be utilized to efficiently and accurately guide the bronchoscopist to the correct subsegmental airway at which point R-EBUS can be used to confirm arrival at the target PPL.

R-EBUS biopsies are performed during bronchoscopy either using moderate sedation or general anesthesia. The use of fluoroscopy during the procedure is operator dependent. Using a reference CT scan the bronchoscope is directed into the appropriate bronchopulmonary segment after which a guide sheath containing a $20 \mathrm{mHz}$ probe is then slowly advanced until visualization of the intended lesion is obtained. The probe is then withdrawn from the sheath, the biopsy instrument is advanced through the sheath, and biopsies are obtained. Published literature results show a range of findings. In one study, histologic diagnosis was confirmed in $69 \%$ of patients using R-EBUS alone, and in $88 \%$ of patients using a combination of R-EBUS and

Electromagnetic Navigational bronchoscopy $(\mathrm{EMN})^{60}$. Results from the AQuIRE Registry failed to show significant contributions with the addition of either R-EBUS or EMN; ${ }^{61}$ while they did show increased diagnostic yield with peripheral TBNA $(47.4 \%)$, it was noted that this particular device was underutilized. The most common cytology needles used for the procedure are either 22 or $21 \mathrm{G}$, but ranging from 19-21 G. As with transthoracic needle aspiration, there are no large studies demonstrating differences in yield rates for different needle gauges.

\section{iii. CT-Guided Transthoracic Needle Aspiration:}

CT-FNA is a minimally invasive procedure that is used to evaluate various lung lesions, particularly peripheral ones. ${ }^{3}$ Transthoracic needle aspiration is able to provide diagnosis of 
peripheral lung lesions with a diagnostic accuracy in the range of $85-95 \%$ in many studies. Its two primary complications are pneumothorax and intraparenchymal bleeding. The procedure involves injecting a local anesthetic from the skin to the pleural surface followed by smooth advancement of the respective needle briskly through the pleural surface into the lung parenchyma and ultimately into the lesion as directed by CT visualization. The procedure may be performed using either intravenous moderate sedation or general anesthesia. Diagnostic rates vary depending upon lesion size and location. Pneumothorax rates are approximately 10-25\% with only a small percentage of patients requiring closed tube thoracostomy. There remains no consensus as to the needle gauge recommended for the procedure. Nearly all comparisons of fine needle aspiration biopsy (FNAB) and cutting needle biopsy have been based only on the capacity to differentiate between benign and malignant lesions. ${ }^{62}$ That same single center retrospective study with a total of 434 consecutive outpatients demonstrated sensitivity, specificity, and accuracy rates for a $22 \mathrm{G}$ Chiba type needle (cytology) of $82.6 \%, 81.3 \%$ and $81.8 \%$ with the respective values for an automated 20 gauge coaxial system (histology) of $93.8 \%, 97.3 \%$ and $95.2 \%$. A variety of cytology needles with gauges ranging from $20-25 \mathrm{G}$ and core biopsy or histological needle gauges ranging from 22-18 G are currently available. With the increasing availability of ROSE; once diagnosis is confirmed by a minimal number of passes, additional passes may be collected for cell block and immunohistochemical stains or mutational analysis. The number of passes needed per procedure has not been defined and may vary based on the availability of ROSE. ${ }^{63}$ Generally at least two passes are recommended.

\section{Guidelines for Use of Rapid On Site Assessment (ROSE)}

\section{A. Overview}

ROSE is an interactive process involving the cytopathology team and the clinical team that performs the FNA. ROSE can influence the effectiveness of FNA in terms of acquiring sufficient tissue for diagnosis and appropriately triaging samples for ancillary tests, as well as by providing immediate direct feedback to the clinical team that performs the image-guided FNA. Collins et al. demonstrated greater efficiency of EBUS-guided FNA procedure along with substantial improvement in health care resource utilization upon the implementation of ROSE. ${ }^{64}$ Ideally, both on-site adequacy and preliminary diagnosis should be included when reporting ROSE. There is limited literature focusing on a detailed protocol for performing ROSE.

While slide preparation may vary slightly among institutions, the most commonly followed procedure is that for each pass, a small portion of the sample is used to produce 2 smeared slides. Positively charged slides are recommended to enhance cellular retention during processing. One smear is used for the ROSE and stained with one of the commercially available fast staining methods. The most commonly used method is The Diff-Quik method on an air dried slide which takes approximately 30 seconds to perform and does not require mounting media and cover slipping for the initial review. The second slide is traditionally fixed with alcohol and stained with the Papanicolaou stain. The needle is then rinsed into a cell collection medium (such as saline, RPMI or CytoLyt) for cell block preparation. Depending on the preliminary diagnostic differential generated from ROSE, other appropriate collecting media may be used for additional samples e.g. culture tubes for microbiology, RPMI for flow cytometry, etc. In the event of a carcinoma diagnosis, additional passes are collected for ancillary testing which include immunohistochemistry to further classify the carcinoma and 
molecular testing. While a cell block is generally favored, smears can also be utilized for these tests if properly validated.

There is no fixed number of needle passes. Instead, the number of passes is determined by the preliminary adequacy, diagnosis, and the need for additional material for potential testing, provided that the patient can tolerate the additional passes. Although ROSE has been shown to reduce the number of recall procedures due to inadequate tissue procurement, ROSE is not a good predictor of an adequate cell block for biomarker and molecular studies.

Documentation of ROSE should include the following information: patient name with two identifiers (e.g. date of birth and medical record number), site of aspiration, size of lesion, total number of needle passes, number of Diff Quik- and Pap-stained slides, choice of cell block/ThinPrep or other concentration technique, any additional material obtained for ancillary studies (i.e. flow cytometry, microbiology), preliminary diagnosis, the clinician who is notified of the preliminary result, names of cytology personnel, and date of the procedure. The ROSE documentation should be incorporated into the final cytology report.

\section{B. ROSE for Sampling Mediastinal Lymph Nodes}

Despite the lack of uniform criteria for adequacy assessment and standardized reporting, there are a few published studies that explored this topic. The University of Minnesota semiquantitatively assessed the number of lymphocytes under $\mathrm{x} 40$ magnification with a score of $0-3$. A score of 0 (<40 lymphocytes) was considered inadequate versus a score of 1 (>40 lymphocytes) or more, alternatively, the presence of clusters of anthracotic pigment-laden macrophages which was considered representative of lymph node sampling and therefore diagnostic. Contamination with bronchial epithelial cells has no influence on adequacy assessment. Samples with malignant cells or granulomas are considered diagnostic regardless of the number of lymphocytes present. Five diagnostic categories were reported, including nondiagnostic, benign, atypical cells, suspicious for carcinoma, and malignant. The data demonstrated that immediate on-site interpretation correlated well with final cytological diagnosis. ${ }^{65}$

However, some found that it is difficult to apply the above scoring criteria to all cases while performing adequacy assessment. Instead, a retrospective study using the same reporting categories and comprising 131 cases of mediastinal lymph node specimens defined samples as non-diagnostic or benign according to the following criteria: 1) Non-diagnostic - no lymphocytes were found in lymph node sampling; 2) Benign - without evidence of tumor, moderate to abundant number of lymphocytes were found in lymph node sampling. ${ }^{66}$ With the aim of defining practical and useful on-site adequacy criteria, another retrospective study established the requirement for adequacy as follows: 1) any smear with $>5$ low power fields (x100 magnification) each containing at least 100 lymphocytes and < 2 groups of bronchial cells; 2) any smear with germinal center fragments; 3) any smear with abnormal results such as malignancy or granuloma. Using this approach, adequacy assessment correlated well with both the final cytological diagnosis and the surgical follow-up. Together with effective cytologistclinician communication, an algorithmic approach to diagnosis and the proposed adequacy criteria can markedly improve the diagnostic yield. ${ }^{67}$ 
To minimize intra- and inter-institutional reporting variability and to establish a standardized ROSE reporting system, Jeffus, et al retrospectively applied the two aforementioned institutional reporting systems to their own study cohort. Their on-site adequacy rates determined by using the two separate systems were almost identical ( $85 \%$ vs. $86 \%)$. The authors recommended a combined modified system, consisting of the adequacy assessment criteria and the tiered diagnostic system. ${ }^{68}$

\section{ROSE for Sampling Lung Masses}

Although the advantages and disadvantages of ROSE for sampling lung lesions have been discussed, few studies describe the adequacy criteria and reporting categories. In practical terms, adequacy criteria for lung mass FNA are self-evident. One study of the role of ROSE for CTguided FNAs of lung nodules mentioned that a smear was considered inadequate if it contained poorly preserved cells or the cellularity was scanty and not compatible with the clinical and/or radiologic impressions. ${ }^{67}$ Similarly, another study defined a specimen as inadequate when no malignant cells or sufficient site-specific tissue is present. ${ }^{69}$ A retrospective study including 64 FNA specimens of lung lesions defined samples as non-diagnostic and benign depending on the absence and presence of bronchial epithelial cells or pigmented macrophages, respectively. A recently published prospective study defined an adequate specimen as one that consisted of adequate numbers of malignant cells or distinct features of granulomatous disease with or without necrosis. $^{70}$

\section{Guidelines For Obtaining, Transporting, and Processing Specimens for Immunochemistry, Flow Cytometry, and Molecular Testing}

Established protocols for handling cytology samples of solid tumors are limited, and the best example is evaluation of HER2 expression in breast cancer. ${ }^{71}$ There is great variation among laboratories for processing non-gynecological cytology. ${ }^{72-74}$ Small biopsies and cytology specimens from patients with lung cancer are often the first, and in some cases, the only available material for diagnostic work-up. ${ }^{75}$ These samples are often used for molecular characterization for predictive markers, which are essential for the selection of appropriate patient therapies $^{76}$ In addition, sub-classification of non-small cell carcinoma into adenocarcinoma versus squamous cell carcinoma has become a necessity for guiding patient management. Therefore, the use of immunocytochemical panels that can aid in the subclassification of NSCLC are an essential tool. ${ }^{77,78}$ When lung cancer is in the differential diagnosis, tissue triage that allows for potential future molecular testing of the diagnostic material should be implemented by each laboratory following multidisciplinary discussions involving pathologists, interventionist, and other clinicians.

Rapid onsite evaluation (ROSE) is encouraged, as it has been demonstrated to reduce the need for subsequent procedures, and it allows for efficient triage of sampled tissues. ${ }^{79}$ During tissue procurement, it is recommended that an effort be made to reduce the number of direct smears produced, thereby preserving more material for subsequent studies. Although many 
laboratories have effectively validated protocols for the use of smears for immunocytochemistry and molecular analysis, this practice is not recommended by the College of American Pathologists (CAP) as the most reliable source of material for molecular testing. ${ }^{80}$ If direct smears are to be used as a source of DNA for molecular studies, additional measures such as digital scanning of original slides to generate a virtual copy are recommended by many legal experts since smears may be the only documentation of disease in some cases of advanced lung carcinoma. When possible, one air dried, modified Diff Quik stained smear or one alcohol fixed, Papanicolaou stained smear should be examined at the time of the procedure to determine adequacy, and the reminder of the aspirate should be placed into a suitable transport liquid for further triage and processing. In addition, core biopsies are sometimes obtained at the time of the FNA to supplement the cytological material. Core biopsies should be placed into fixative (buffered formalin) and transported to the laboratory for histologic processing.

When the diagnosis of lung cancer is suspected or confirmed during ROSE, the aspirates should ideally be fixed in alcohol or buffered formalin until further processing. The use of alcohol is generally preferred for material that is going to be used for molecular testing since the yield of nucleic acid is greater with less degradation artifacts. However, alcohol fixation can present problems for immunocytochemical analysis. The immunohistochemical protocols developed for formalin-fixed tissue may not apply completely to alcohol fixed material. Thus, requiring validation of each antibody for alcohol-fixed tissue. In addition, there are some antibodies that are less effective when applied to alcohol fixed-tissue even after rigorous validation attempts. ${ }^{81}$ More importantly, improvements in the ability to extract DNA from formalin-fixed paraffin-embedded (FFPE) tissue have been made.

When a lymphoproliferative lesion is suspected during triage of the specimen, unfixed material for flow cytometry should be obtained. In these cases, the unfixed sample can be collected and transported in an isotonic fluid such as cell culture medium (RPMI for example) or enriched saline (Hank's solution). If needed for diagnostic purposes, an aliquot of the material can be used to produce a liquid-based preparation, but adequate material should be reserved for flow cytometric analysis. An alternative protocol is to split the obtained material for flow cytometry and an FFPE cell block that can be used for further classification of the lymphoid cells typically by immunohistochemical evaluation.

When additional dedicated aspirates are deemed necessary for ancillary studies, this should be communicated to the interventionist. If further passes are obtained, it is important to check each pass for adequacy.

Cell block preparations are an important tool for tumor analysis, and they provide a means of preserving tissue for long-term storage and for future discoveries. In addition, cell block preparations are the recommended method by CAP for molecular studies ${ }^{80}$. Cell blocks allow for quality assurance and control, since the number and quality of the neoplastic cells can be estimated as well as the presence of other cell types that may influence subsequent analysis. For molecular studies, the number of tumor cells and the amount of tumor nuclei in the preparation are essential components for an adequate performance. The presence of necrotic debris and number of contaminating lymphocytes can influence results. Most molecular techniques can now detect allelic alteration in less than 5\% of tumor nuclei, but if the majority of DNA comes from benign lymphocytes and the molecular alteration is present at a low rate in tumor cells, a false negative result could result. Several studies have assessed the minimum 
number of tumor cells required for evaluation. Although the number depends upon the molecular test preformed, a general estimate is a minimum number of 2000 tumor cells, and the tumor content should be greater than $20 \% .^{82-84}$

\section{Conclusion:}

The use of cytology in the diagnosis of pulmonary disease and in particular pulmonary cancer has increased tremendously especially in the era of personalized therapy. In that respect, diagnosis of lung cancer by cytology especially TBNA has been the prototype for many future applications to other cancers. This series of publications are focused on establishing an in depth understanding of the different cytology sampling techniques, their indications, methodology and handling the collected specimens. The additional application of ancillary techniques such as immunocytotochemistry and molecular testing mandates that the limited cytology samples be efficiently utilized to maximize the results and avoid additional more invasive sampling methods. It is logical to anticipate that future publications will focus on standardizing the processing methodology for DNA and RNA retrieval and for building proper biorepositories. 


\section{References:}

1. Layfield LJ, Baloch Z, Elsheikh T, et al. Standardized terminology and nomenclature for respiratory cytology: The Papanicolaou Society of Cytopathology guidelines. Diagnostic cytopathology. May 2016;44(5):399-409.

2. Layfield LJ, Roy-Chowdhuri S, Baloch Z, et al. Utilization of ancillary studies in the cytologic diagnosis of respiratory lesions: The papanicolaou society of cytopathology consensus recommendations for respiratory cytology. Diagnostic cytopathology. Dec 2016;44(12):10001009.

3. Michael CW, Hoda RS, Saqi A, et al. Committee I: Indications for pulmonary cytology sampling methods. Diagnostic cytopathology. Dec 2016;44(12):1010-1023.

4. Rivera MP, Mehta AC, Wahidi MM. Establishing the diagnosis of lung cancer: Diagnosis and management of lung cancer, 3rd ed: American College of Chest Physicians evidence-based clinical practice guidelines. Chest. May 2013;143(5 Suppl):e142S-165S.

5. Uke MS, Pathuthara S, Shaikh A, Kumar R, Kane S. Is the Morning Sputum Sample Superior to the Fresh Sputum Sample for the Detection of Malignant Cells? Acta Cytol. 2017;61(3):223-229.

6. Koss LG. The Cytological Diagnosis of Cancer. Geriatrics. Oct 1964;19:745-759.

7. Jones DP. Diagnostic work-up of chest disease. The Surgical clinics of North America. Aug 1980;60(4):743-755.

8. Healy TM, Borrie J. Lung cancer diagnosis: evaluation of diagnostic techniques. The New Zealand medical journal. May 14 1975;81(539):423-424.

9. Gilpin C, Kim SJ, Lumb R, Rieder HL, Van Deun A, Working Group on Sputum Smear M. Critical appraisal of current recommendations and practices for tuberculosis sputum smear microscopy. The international journal of tuberculosis and lung disease : the official journal of the International Union against Tuberculosis and Lung Disease. Sep 2007;11(9):946-952.

10. Murray MP, Doherty CJ, Govan JR, Hill AT. Do processing time and storage of sputum influence quantitative bacteriology in bronchiectasis? Journal of medical microbiology. Jul 2010;59(Pt 7):829-833.

11. Ho J, Marks GB, Fox GJ. The impact of sputum quality on tuberculosis diagnosis: a systematic review. The international journal of tuberculosis and lung disease : the official journal of the International Union against Tuberculosis and Lung Disease. May 2015;19(5):537-544.

12. Saraswathy Veena V, Sara George P, Jayasree K, Sujathan K. Comparative analysis of cell morphology in sputum samples homogenized with dithiothreitol, $\mathrm{N}$-acetyl-L cysteine, Cytorich $((R))$ red preservative and in cellblock preparations to enhance the sensitivity of sputum cytology for the diagnosis of lung cancer. Diagnostic cytopathology. Jul 2015;43(7):551-558.

13. Choi YD, Han CW, Kim JH, et al. Effectiveness of sputum cytology using ThinPrep method for evaluation of lung cancer. Diagnostic cytopathology. Mar 2008;36(3):167-171.

14. Wu GP, Wang EH, Li JH, Fu ZM, Han S. Clinical application of the liquid-based cytological test in cytological screening of sputum for the diagnosis of lung cancer. Respirology. Jan 2009;14(1):124-128.

15. Agusti C, Xaubet A, Monton C, et al. Induced sputum in the diagnosis of peripheral lung cancer not visible endoscopically. Respiratory medicine. Oct 2001;95(10):822-828.

16. Bandyopadhyay T, Gerardi DA, Metersky ML. A comparison of induced and expectorated sputum for the microbiological diagnosis of community acquired pneumonia. Respiration; international review of thoracic diseases. 2000;67(2):173-176. 
17. Paggiaro PL, Chanez P, Holz O, et al. Sputum induction. The European respiratory journal. Supplement. Sep 2002;37:3s-8s.

18. Fishman JA, Roth RS, Zanzot E, Enos EJ, Ferraro MJ. Use of induced sputum specimens for microbiologic diagnosis of infections due to organisms other than Pneumocystis carinii. Journal of clinical microbiology. Jan 1994;32(1):131-134.

19. Peter JG, Theron G, Pooran A, Thomas J, Pascoe M, Dheda K. Comparison of two methods for acquisition of sputum samples for diagnosis of suspected tuberculosis in smear-negative or sputum-scarce people: a randomised controlled trial. The Lancet. Respiratory medicine. Aug 2013;1(6):471-478.

20. Al Zahrani K, Al Jahdali H, Poirier L, Rene P, Menzies D. Yield of smear, culture and amplification tests from repeated sputum induction for the diagnosis of pulmonary tuberculosis. The international journal of tuberculosis and lung disease : the official journal of the International Union against Tuberculosis and Lung Disease. Sep 2001;5(9):855-860.

21. Chang KC, Leung CC, Yew WW, Tam CM. Supervised and induced sputum among patients with smear-negative pulmonary tuberculosis. The European respiratory journal. May 2008;31(5):1085-1090.

22. Souza Pinto V, Bammann RH. Chest physiotherapy for collecting sputum samples from HIVpositive patients suspected of having tuberculosis. The international journal of tuberculosis and lung disease : the official journal of the International Union against Tuberculosis and Lung Disease. Dec 2007;11(12):1302-1307.

23. Efthimiadis A, Jayaram L, Weston S, Carruthers S, Hargreave FE. Induced sputum: time from expectoration to processing. The European respiratory journal. Apr 2002;19(4):706-708.

24. Malekmohammad M, Marjani M, Tabarsi P, et al. Diagnostic yield of post-bronchoscopy sputum smear in pulmonary tuberculosis. Scandinavian journal of infectious diseases. May 2012;44(5):369-373.

25. George PM, Mehta M, Dhariwal J, et al. Post-bronchoscopy sputum: improving the diagnostic yield in smear negative pulmonary TB. Respiratory medicine. Nov 2011;105(11):1726-1731.

26. Aderaye G, H GE, Aseffa A, Worku A, Lindquist L. Comparison of acid-fast stain and culture for Mycobacterium tuberculosis in pre- and post-bronchoscopy sputum and bronchoalveolar lavage in HIV-infected patients with atypical chest X-ray in Ethiopia. Annals of thoracic medicine. Oct 2007;2(4):154-157.

27. Lim GH, Ross $\mathrm{P}$, Landman S. Bronchial brush biopsy and primary lung carcinoma. The Medical journal of Australia. Aug 9 1975;2(6):207-210.

28. Zavala DC, Richardson RH, Mukerjee PK, Rossi NP, Bedell GN. Use of the bronchofiberscope for bronchial brush biopsy. Diagnostic results and comparison with other brushing techniques. Chest. Jun 1973;63(6):889-892.

29. Sato M, Saito Y, Nagamoto N, et al. Diagnostic value of differential brushing of all branches of the bronchi in patients with sputum positive or suspected positive for lung cancer. Acta Cytol. Nov-Dec 1993;37(6):879-883.

30. Meyer KC, Raghu G, Baughman RP, et al. An official American Thoracic Society clinical practice guideline: the clinical utility of bronchoalveolar lavage cellular analysis in interstitial lung disease. American journal of respiratory and critical care medicine. May 1 2012;185(9):10041014.

31. Orholm M, Lundgren JD, Nielsen TL, Iversen J. Indication for fiberoptic bronchoscopy in HIVinfected patients suspected for Pneumocystis carinii pneumonia. Danish medical bulletin. Feb 1990;37(1):86-89.

This article is protected by copyright. All rights reserved. 
32. Technical recommendations and guidelines for bronchoalveolar lavage (BAL). Report of the European Society of Pneumology Task Group. The European respiratory journal. Jun 1989;2(6):561-585.

33. Radhakrishna N, Farmer M, Steinfort DP, King P. A Comparison of Techniques for Optimal Performance of Bronchoalveolar Lavage. Journal of bronchology \& interventional pulmonology. Oct 2015;22(4):300-305.

34. Izumo T, Sasada S, Chavez C, Matsumoto Y, Hayama M, Tsuchida T. The diagnostic value of histology and cytology samples during endobronchial ultrasound with a guide sheath. Japanese journal of clinical oncology. Apr 2015;45(4):362-366.

35. Meyer KC, Raghu G. Bronchoalveolar lavage for the evaluation of interstitial lung disease: is it clinically useful? The European respiratory journal. Oct 2011;38(4):761-769.

36. Baughman RP. Technical aspects of bronchoalveolar lavage: recommendations for a standard procedure. Seminars in respiratory and critical care medicine. Oct 2007;28(5):475-485.

37. Hurter T, Hanrath P. Endobronchial sonography: feasibility and preliminary results. Thorax. Jul 1992;47(7):565-567.

38. Kurimoto N, Murayama M, Yoshioka S, Nishisaka T, Inai K, Dohi K. Assessment of usefulness of endobronchial ultrasonography in determination of depth of tracheobronchial tumor invasion. Chest. Jun 1999;115(6):1500-1506.

39. Yasufuku K, Chiyo M, Koh E, et al. Endobronchial ultrasound guided transbronchial needle aspiration for staging of lung cancer. Lung cancer. Dec 2005;50(3):347-354.

40. Berania I, Kazakov J, Khereba M, et al. Endoscopic Mediastinal Staging in Lung Cancer Is Superior to "Gold Standard" Surgical Staging. Ann Thorac Surg. Feb;101(2):547-550.

41. Eberhardt R, Kahn N, Gompelmann D, Schumann M, Heussel CP, Herth FJ. LungPoint--a new approach to peripheral lesions. J Thorac Oncol. Oct;5(10):1559-1563.

42. Zhang W, Chen S, Dong X, Lei P. Meta-analysis of the diagnostic yield and safety of electromagnetic navigation bronchoscopy for lung nodules. J Thorac Dis. May;7(5):799-809.

43. Chen A, Chenna P, Loiselle A, Massoni J, Mayse M, Misselhorn D. Radial probe endobronchial ultrasound for peripheral pulmonary lesions. A 5-year institutional experience. Ann Am Thorac Soc. May;11(4):578-582.

44. Cerfolio RJ, Bryant AS, Eloubeidi MA. Accessing the aortopulmonary window (\#5) and the paraaortic (\#6) lymph nodes in patients with non-small cell lung cancer. The Annals of thoracic surgery. Sep 2007;84(3):940-945.

45. Dhooria S, Aggarwal AN, Gupta D, Behera D, Agarwal R. Utility and Safety of Endoscopic Ultrasound With Bronchoscope-Guided Fine-Needle Aspiration in Mediastinal Lymph Node Sampling: Systematic Review and Meta-Analysis. Respiratory care. Jul 2015;60(7):1040-1050.

46. Edey AJ, Pollentine A, Doody C, Medford AR. Differentiating benign from malignant mediastinal lymph nodes visible at EBUS using grey-scale textural analysis. Respirology. Apr;20(3):453-458.

47. Fujiwara T, Yasufuku K, Nakajima T, et al. The utility of sonographic features during endobronchial ultrasound-guided transbronchial needle aspiration for lymph node staging in patients with lung cancer: a standard endobronchial ultrasound image classification system. Chest. Sep;138(3):641-647.

48. Gupta NC, Tamim WJ, Graeber GG, Bishop HA, Hobbs GR. Mediastinal lymph node sampling following positron emission tomography with fluorodeoxyglucose imaging in lung cancer staging. Chest. Aug 2001;120(2):521-527.

49. Li S, Zheng $Q, M a ~ Y$, et al. Implications of false negative and false positive diagnosis in lymph node staging of NSCLC by means of (1)(8)F-FDG PET/CT. PloS one. 2013;8(10):e78552.

50. Diacon AH, Schuurmans MM, Theron J, et al. Transbronchial needle aspirates: how many passes per target site? The European respiratory journal. Jan 2007;29(1):112-116. 
51. Kinsey CM, Arenberg DA. Endobronchial ultrasound-guided transbronchial needle aspiration for non-small cell lung cancer staging. Am J Respir Crit Care Med. Mar 15;189(6):640-649.

52. Yarmus L, Akulian J, Gilbert C, et al. Optimizing endobronchial ultrasound for molecular analysis. How many passes are needed? Ann Am Thorac Soc. Dec;10(6):636-643.

53. Oki M, Saka H, Kitagawa C, et al. Randomized Study of 21-gauge Versus 22-gauge Endobronchial Ultrasound-guided Transbronchial Needle Aspiration Needles for Sampling Histology Specimens. J Bronchology Interv Pulmonol. Oct;18(4):306-310.

54. Saji J, Kurimoto N, Morita K, et al. Comparison of 21-gauge and 22-gauge Needles for Endobronchial Ultrasound-Guided Transbronchial Needle Aspiration of Mediastinal and Hilar Lymph Nodes. J Bronchology Interv Pulmonol. Jul;18(3):239-246.

55. Yarmus LB, Akulian J, Lechtzin N, et al. Comparison of 21-gauge and 22-gauge aspiration needle in endobronchial ultrasound-guided transbronchial needle aspiration: results of the American College of Chest Physicians Quality Improvement Registry, Education, and Evaluation Registry. Chest. Apr;143(4):1036-1043.

56. Xing J, Manos S, Monaco SE, Wilson DO, Pantanowitz L. Endobronchial Ultrasound-Guided Transbronchial Needle Aspiration: A Pilot Study to Evaluate the Utility of the ProCore Biopsy Needle for Lymph Node Sampling. Acta Cytol. 2016;60(3):254-259.

57. Tyan C, Patel P, Czarnecka K, et al. Flexible 19-Gauge Endobronchial Ultrasound-Guided Transbronchial Needle Aspiration Needle: First Experience. Respiration; international review of thoracic diseases. 2017;94(1):52-57.

58. Rivera MP, Mehta AC, Wahidi MM. Establishing the diagnosis of lung cancer: Diagnosis and management of lung cancer, 3rd ed: American College of Chest Physicians evidence-based clinical practice guidelines. Chest. May 2013;143(5 Suppl):e142S-e165S.

59. Michaelson ED, Serafind SM. Quantitative differences in the cellular yield of two bronchial brushes. The American review of respiratory disease. Aug 1975;112(2):267-268.

60. Eberhardt R, Anantham D, Ernst A, Feller-Kopman D, Herth F. Multimodality bronchoscopic diagnosis of peripheral lung lesions: a randomized controlled trial. American journal of respiratory and critical care medicine. Jul 1 2007;176(1):36-41.

61. Ost DE, Ernst A, Lei X, et al. Diagnostic Yield and Complications of Bronchoscopy for Peripheral Lung Lesions. Results of the AQuIRE Registry. American journal of respiratory and critical care medicine. Jan 1 2016;193(1):68-77.

62. Guimaraes MD, Marchiori E, Hochhegger B, Chojniak R, Gross JL. CT-guided biopsy of lung lesions: defining the best needle option for a specific diagnosis. Clinics. 2014;69(5):335-340.

63. Manhire A, Charig M, Clelland C, et al. Guidelines for radiologically guided lung biopsy. Thorax. Nov 2003;58(11):920-936.

64. Collins BT, Chen AC, Wang JF, Bernadt CT, Sanati S. Improved laboratory resource utilization and patient care with the use of rapid on-site evaluation for endobronchial ultrasound fine-needle aspiration biopsy. Cancer cytopathology. Oct 2013;121(10):544-551.

65. Alsharif M, Andrade RS, Groth SS, Stelow EB, Pambuccian SE. Endobronchial ultrasound-guided transbronchial fine-needle aspiration: the University of Minnesota experience, with emphasis on usefulness, adequacy assessment, and diagnostic difficulties. American journal of clinical pathology. Sep 2008;130(3):434-443.

66. Feller-Kopman D, Yung RC, Burroughs F, Li QK. Cytology of endobronchial ultrasound-guided transbronchial needle aspiration: a retrospective study with histology correlation. Cancer. Dec 25 2009;117(6):482-490.

67. Nayak A, Sugrue C, Koenig S, Wasserman PG, Hoda S, Morgenstern NJ. Endobronchial ultrasound-guided transbronchial needle aspirate (EBUS-TBNA): a proposal for on-site adequacy criteria. Diagnostic cytopathology. Feb 2012;40(2):128-137. 
68. Jeffus SK, Joiner AK, Siegel ER, et al. Rapid on-site evaluation of EBUS-TBNA specimens of lymph nodes: Comparative analysis and recommendations for standardization. Cancer cytopathology. Jun 2015;123(6):362-372.

69. Thiryayi SA, Rana DN, Narine N, Najib M, Bailey S. Establishment of an endobronchial ultrasound-guided transbronchial fine needle aspiration service with rapid on-site evaluation: 2 years experience of a single UK centre. Cytopathology : official journal of the British Society for Clinical Cytology. Feb 252016.

70. Mfokazi A, Wright CA, Louw M, et al. Direct comparison of liquid-based and smear-based cytology with and without rapid on-site evaluation for fine needle aspirates of thoracic tumors. Diagnostic cytopathology. May 2016;44(5):363-368.

71. Wolff AC, Hammond ME, Hicks DG, et al. Recommendations for human epidermal growth factor receptor 2 testing in breast cancer: American Society of Clinical Oncology/College of American Pathologists clinical practice guideline update. Archives of pathology \& laboratory medicine. Feb 2014;138(2):241-256.

72. Moriarty AT, Nayar R, Auger M, et al. Nongynecologic cytology practice patterns: a survey of participants in the College of American Pathologists interlaboratory comparison program in nongynecologic cytopathology. Archives of pathology \& laboratory medicine. Jul 2014;138(7):885-889.

73. Fischer AH, Schwartz MR, Moriarty AT, et al. Immunohistochemistry practices of cytopathology laboratories: a survey of participants in the College of American Pathologists Nongynecologic Cytopathology Education Program. Archives of pathology \& laboratory medicine. Sep 2014;138(9):1167-1172.

74. Sturgis CD, Marshall CB, Barkan GA, et al. Respiratory Cytology--Current Trends Including Endobronchial Ultrasound-Guided Biopsy and Electromagnetic Navigational Bronchoscopy: Analysis of Data From a 2013 Supplemental Survey of Participants in the College of American Pathologists Interlaboratory Comparison Program in Nongynecologic Cytology. Archives of pathology \& laboratory medicine. Jan 2016;140(1):22-28.

75. Molina JR, Adjei AA, Jett JR. Advances in chemotherapy of non-small cell lung cancer. Chest. Oct 2006;130(4):1211-1219.

76. Moreira AL, Eng J. Personalized therapy for lung cancer. Chest. Dec 2014;146(6):1649-1657.

77. Rekhtman N, Ang DC, Sima CS, Travis WD, Moreira AL. Immunohistochemical algorithm for differentiation of lung adenocarcinoma and squamous cell carcinoma based on large series of whole-tissue sections with validation in small specimens. Modern pathology: an official journal of the United States and Canadian Academy of Pathology, Inc. Oct 2011;24(10):1348-1359.

78. Travis WD, Rekhtman N, Riley GJ, et al. Pathologic diagnosis of advanced lung cancer based on small biopsies and cytology: a paradigm shift. Journal of thoracic oncology : official publication of the International Association for the Study of Lung Cancer. Apr 2010;5(4):411-414.

79. van der Heijden EH, Casal RF, Trisolini R, et al. Guideline for the acquisition and preparation of conventional and endobronchial ultrasound-guided transbronchial needle aspiration specimens for the diagnosis and molecular testing of patients with known or suspected lung cancer. Respiration; international review of thoracic diseases. 2014;88(6):500-517.

80. Lindeman NI, Cagle PT, Beasley MB, et al. Molecular testing guideline for selection of lung cancer patients for EGFR and ALK tyrosine kinase inhibitors: guideline from the College of American Pathologists, International Association for the Study of Lung Cancer, and Association for Molecular Pathology. Journal of thoracic oncology : official publication of the International Association for the Study of Lung Cancer. Jul 2013;8(7):823-859.

This article is protected by copyright. All rights reserved. 
81. Sauter JL, Grogg KL, Vrana JA, Law ME, Halvorson JL, Henry MR. Young investigator challenge: Validation and optimization of immunohistochemistry protocols for use on cellient cell block specimens. Cancer cytopathology. Feb 2016;124(2):89-100.

82. Roy-Chowdhuri S, Goswami RS, Chen $\mathrm{H}$, et al. Factors affecting the success of next-generation sequencing in cytology specimens. Cancer cytopathology. Nov 2015;123(11):659-668.

83. Cannonier SA, Sterling JA. The Role of Hedgehog Signaling in Tumor Induced Bone Disease. Cancers. Aug 26 2015;7(3):1658-1683.

84. Roy-Chowdhuri S, Chow CW, Kane MK, et al. Optimizing the DNA yield for molecular analysis from cytologic preparations. Cancer cytopathology. Apr 2016;124(4):254-260.

This article is protected by copyright. All rights reserved. 


\section{Figure Legends}

Figure 1. The mediastinal and hilar lymph nodes generally accessible via EBUS bronchoscopy. A) AP coronal view and B) right-sided sagittal view of the mediastinum and hila.

Figure 2. A) Enlarged subcarinal (7) lymph node seen on cross-sectional CT image. B) Level 7 lymph node undergoing real-time EBUS TBNA.

Figure 3. Anatomy of the EBUS bronchoscope tip with viewing angles.

This article is protected by copyright. All rights reserved. 